\title{
On the use of Raman and FTIR Spectroscopy for the Analysis of Silica-based Nanofillers
}

\author{
C. Yeung* ${ }^{1}$, G. Gherbaz ${ }^{1}$ and A. S. Vaughan ${ }^{1}$ \\ ${ }^{1}$ University of Southampton, UK \\ *E-mail: cy3g09@ecs.soton.ac.uk
}

The potential of polymeric nanocomposites as solid insulation systems has been a topic of great discussion. Although this approach as a means to engineering materials with improved properties has been well established, many of the fundamentals aspects of this class of materials remains poorly understood. For example, the long term dielectric characteristics of so-called nanodielectrics is one of these topics. Whilst the nature of the interfacial region within such systems is believed to be key in determining performance, further investigation is required in order better to understand the macroscopic behaviour of nanocomposites. Such studies are vital for fundamental change, bringing an alternative to conventional polymers and filled composites and so making a massive impact on industry.

This paper concerns interfaces in nanodielectrics and sets out to explore the effect of quantified changes in surface functionalisation. Specifically, we have used vibrational spectroscopy to examine and characterise the relevant effects of modifying the surface chemistry of nanosilica with commercial silane methods. Confocal Raman spectroscopy is used to provide qualitative data concerning the functionalization level, whilst Fourier Transform Infrared spectroscopy is used to provide more quantitative data. In this paper, we present the first step towards the design and quantification of nanoparticle surface chemistry - a step that we believe will ultimately allow the interphase to be optimized to meet demanding dielectric requirements. 